\title{
Los atractores de viajes como concepto operacional en el estudio de la movilidad urbana
}

\section{Laura Cristina Aón}

Instituto de Investigaciones y Políticas del Ambiente Construido, CONICET / Universidad Nacional de La Plata, Argentina. https://orcid.org/oooo-0002-8155-7091

\section{María Luciana Giglio}

Instituto de Investigaciones y Políticas del Ambiente Construido, CONICET / Universidad Nacional de La Plata, Argentina.

https://orcid.org/oooo-0002-7306-4043

\section{Nadia Silvana Freaza}

Instituto de Investigaciones y Políticas del Ambiente Construido, CONICET / Universidad Nacional de La Plata, Argentina. https://orcid.org/oooo-0003-4503-3458

\section{Cristian Agustín Cola}

Instituto de Investigaciones en Energía No Convencional, CONICET / Universidad Nacional de Salta, Argentina.

https://orcid.org/oooo-0002-7065-7062

\section{María Julieta López}

Instituto de Investigaciones y Políticas del Ambiente Construido, CONICET / Universidad Nacional de La Plata, Argentina.

https://orcid.org/oooo-0002-7472-0604

Recibido: 16 de abril de 2019. Aceptado: 29 de octubre de 2019.

\section{Resumen}

Esta comunicación expone las estrategias y técnicas empleadas para los relevamientos y el procesamiento de encuestas de movilidad en atractores de viaje, desarrolladas en el periodo 2013-2017 en la ciudad de La Plata, cuyo propósito fue investigar los patrones de movilidad de la ciudad. El trabajo de campo debe su origen al marco teórico de la tesis doctoral "Racionalidad no económica de la movilidad urbana de pasajeros" aplicado en el contexto del proyecto PPID U002++. El concepto de atractor de viajes forma 
parte de la teoría de la generación de viajes (ITE, 1991) y se define como lugares, áreas o zonas donde convergen actividades no residenciales que atraen viajes de pasajeros. El artículo expone el proceso de elaboración y procesamiento de encuestas y las sucesivas adaptaciones metodológicas realizadas para la investigación. Las principales conclusiones se desarrollan en torno a lo conceptual y lo metodológico señalando, por un lado, la importancia de poner en valor el estudio de atractores para el abordaje integral de la ciudad y la movilidad y, por otro lado, resaltando el valor de la construcción colectiva de conocimiento en la operacionalización de los trabajos de campo.

Palabras Clave: Atractores De Viaje. Encuestas De Movilidad. Metodologías.

\title{
Travel attractors as an operational concept in the study of urban mobility
}

\begin{abstract}
This communication exposes the strategies and techniques used for surveys and processing of mobility surveys in travel attractors, developed in the 2013-2017 period in the city of La Plata, whose purpose was to investigate the city's mobility patterns. The field work owes its origin to the theoretical framework of the doctoral thesis "Noneconomic rationality of urban passenger mobility" applied in the context of the PPID U002 project1. The concept of travel attractor is part of the theory of travel generation (ITE, 1991) and is defined as places, areas or areas where non-residential activities converge that attract passenger trips. The article exposes the process of elaboration and processing of surveys and the successive methodological adaptations made for research. The main conclusions are developed around the conceptual and methodological aspects, pointing out, on the one hand, the importance of valuing the study of attractors for the integral approach of the city and mobility and, on the other hand, highlighting the value of the collective construction of knowledge in the operationalization of field work.
\end{abstract}

Key words: Travel Attractors. Mobility Surveys. Methodologies.

Palavras-chave: Atratores de viagens. Pesquisas de mobilidade. Metodologias.

\section{Introducción}

Estudiar la movilidad en ciudades grandes e intermedias, constituye un desafío de alta complejidad. La magnitud de los desplazamientos urbanos y su naturaleza cambiante e irregular, se acentúan dentro de los contextos latinoamericanos, reconocidos por ser socialmente desiguales, espacialmente extensos y económicamente inestables. Más allá de su multidimensionalidad y multicausalidad estructural, el enjambre de problemas que conlleva la movilidad, demanda la formulación de nuevas preguntas. El presente trabajo toma como punto de partida la siguiente interrogante: ¿Cómo abordar la diversidad y complejidad de la movilidad urbana actual, mediante qué herramientas conceptuales y metodológicas?

El Observatorio de Movilidad del Gran La Plata (LII - IIPAC-CONICET/UNLP), como espacio de investigación colectivo, fue concebido como una nueva herramienta conceptual y metodológica para el estudio de la movilidad. Su particularidad radica en poner en valor las potencialidades de los conceptos e instrumentos clásicos preexistentes reformulándolos y combinándolos, con el objetivo de desarrollar nuevas formas (más ágiles, flexibles. extensivas y por qué no, más económicas) de construir información de movilidad cuantitativa y cualitativa. 
El Observatorio tuvo desde sus inicios, el propósito de extender las búsquedas de los estudios de movilidad clásicos, desde el "qué", "cómo", "cuándo", "cuánto" y "dónde", hasta el "quiénes" y "por qué", dimensiones que las ciencias sociales reclaman hace tiempo a las ciencias aplicadas. El marco conceptual de partida para estas búsquedas, es la teoría de la generación de viajes, expresada en el "Manual of trip generation" (ITE, 1991) que pone en relación los enfoques analíticos de la producción y la atracción de viajes. El enfoque conceptual cuantitativo tuvo un mayor desarrollo desde la década de 1960, cuando el avance tecnológico permitió comenzar a modelizar y simular en ordenadores la dinámica de movilidad en ciudades (Alonso, 1964; Bates, 1988; Bruton, 1970; Ortuzar y Wiliumsenn, 1994). Estos enfoques, espaciales y cuantitativos, expresan su alcance social y económico en la construcción de los modelos de generación basados en clasificación de grupos, familias y hogares, con lo cual tienen una capacidad explicativa superior al del tradicional binomio origen-destino de los estudios clásicos. De esta forma se admite de manera consistente la doble mirada de los viajes, es decir, la lectura analítica de la producción de viajes y la lectura analítica de la atracción de esos mismos viajes.

Basándose en este enfoque, originario del marco teórico metodológico de la tesis doctoral "Racionalidad no económica de la movilidad urbana de pasajeros", y en la búsqueda por optimizar el trabajo de campo en los estudios de movilidad, el Observatorio desarrolló una serie de encuestas en la ciudad de La Plata. Dicha experiencia de aplicación, comenzó a desarrollarse en el año 2013, en un proceso continuado de relevamientos, procesamientos y evaluaciones (Aón et al., 2017). Las prácticas desarrolladas por el Observatorio combinan estrategias metodológicas tradicionales con nuevas tecnologías, tomando como una fuente de información clave y como unidad de análisis territorial a los "atractores de viaje". A partir de dicha unidad, el objetivo de los relevamientos fue la construcción de datos sinérgicos de producción-atracción de viajes capturando, por un lado, datos clave de las componentes de la producción de los viajes (del hogar donde se producen las movilidades) y, por otro lado, del atractor de viaje donde convergen espacio temporalmente, las movilidades y miembros de los diferentes hogares de muy diversos puntos de la ciudad.

En este marco el presente artículo recapitula los trabajos de campo realizados en atractores de viajes para la ciudad de La Plata en el periodo 2013-2017, recorriendo las diferentes etapas de diseño metodológico y operativo, ejecución del relevamiento y procesamiento de las encuestas de movilidad. A modo de relato detallado, se pretende visibilizar, por un lado, el valor metodológico en la exposición del proceso de evolución operativa y de las sucesivas estrategias que fueron desplegadas para alcanzar mejores resultados. Por otro lado, se pretende dar cuenta del potencial del Observatorio como herramienta conceptual y operacional colectiva para el avance de las investigaciones tanto individuales como grupales, las cuales permiten retroalimentar y complejizar tanto las herramientas como las preguntas de investigación acerca de la movilidad y la ciudad.

\section{El atractor de viajes como concepto operacional}

El concepto de "atractor de viajes" pertenece a la literatura del transporte y es parte de un sistema conceptual más complejo, el de la "generación de viajes". Este concepto explica el binomio producción/atracción de viajes y que constituyen una base conceptual presente en la mayoría de los estudios de transporte realizados en ciudades argentinas desde los años ' 80 .

Efectivamente, en nuestro país se han aplicado históricamente y hasta hace pocos años, estudios de transporte urbano cuantitativos basados en encuestas de origen y destino 
de viajes (PTUMA, 2008, 2009a, 2009b, 2010a, 2010b, 2012a, 2012b, 2012c; UNC, 2000; UNCo, 1992; UNCuyo, 1986, 1998; UNLP, 1993, 1998; UNR 1993, 2002; UNS, 2014). Este conjunto de estudios está basado en encuestas domiciliarias tradicionales, mediante las cuáles se construyen muestras de gran escala (entre un 1 y 1,5\% de la población urbana), dado que persiguen un criterio de representatividad estadística y territorial de población y de hogares para cada ciudad estudiada.

La metodología de encuestas domiciliarias está basada en la modelización urbana del transporte y se apoya en la teoría de la generación de viajes, formulada en "Manual of trip generation" (ITE, 1991) la cual enfoca el análisis de transporte (todos los viajes) en la unidad-hogar, clasificando los viajes según dos criterios: "viajes basados en hogar" (HB) y "viajes no basados en hogar" (NHB). Los HB tienen al hogar en alguno de los extremos del viaje (origen o destino). Los NHB no se originan ni se destinan al hogar. A partir de estos conceptos, se asume que la generación de viajes de pasajeros es una función de los atributos sociales y económicos de los hogares donde se definen las actividades que los miembros del hogar desarrollan, lo cual también estará sujeto al lugar donde se ubique su vivienda.

En este marco se establecen los conceptos de "producción" y "atracción", como componentes de la generación de viajes, donde la producción constituye una lectura analítica de los viajes en relación al uso residencial y al hogar, mientras que la atracción es una lectura analítica de esos mismos viajes, pero desde la perspectiva de usos noresidenciales, donde las personas realizan sus actividades fuera del hogar. Es decir, aquellas que, precisamente, producen movilidad y demandan transporte: los atractores de viaje (Figura 1).

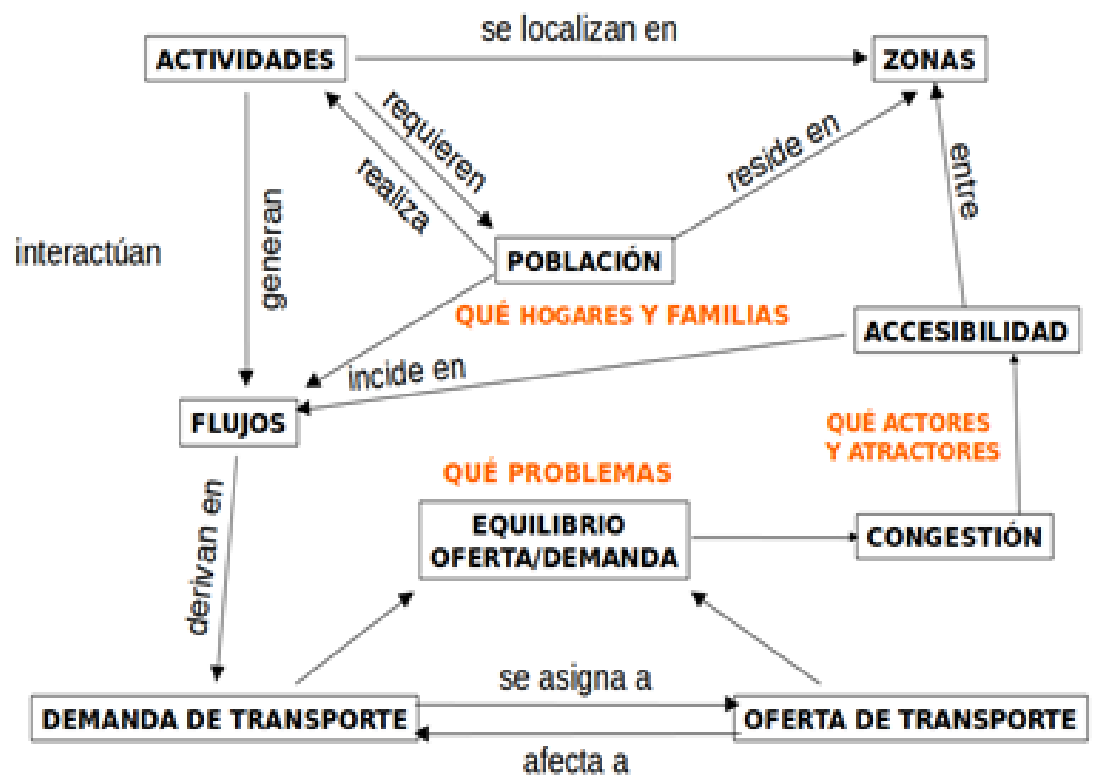

Figura 1. Esquema conceptual de estudios cuantitativos de transporte. Elaboración: Laura Aón. Fuente: Avances de tesis doctoral "Racionalidad no económica de la movilidad urbana de pasajeros".

La formulación conceptual de la generación, producción y atracción de viajes, está orientada a construir diferentes miradas analíticas de los viajes y de la movilidad, útiles para la problematización adecuada e intervención posterior precisa, con políticas de oferta de demanda e incluso, de desarrollo urbano. Estos conceptos operacionales de la teoría clásica de transporte, permiten saber: (i) qué problemas existen (cantidad de 
hogares con dependencia de modos de transporte motorizados, usos no residenciales que atraen más viajes de los que pueden contener, entre otros); (ii) dónde están localizados dichos problemas (en qué zonas o barrios, qué arterias o rutas, a qué oferta de transporte público responden o con qué otros problemas de naturaleza espacial se solapan) y (iii) cuáles son los actores que están involucrados (tipos de hogares, establecimientos de servicios públicos o privados, centros de abastecimiento) (Figura 1).

Este abordaje es clave para las aproximaciones cuantitativas, que son las que permiten el anclaje espacial de la movilidad en la ciudad. En este enfoque, estudiar la movilidad de pasajeros con propósitos de intervención necesita del análisis y componentes espaciales. Sin embargo, la tradición de estudios de origen y destino de viajes, ha reforzado la separación de la comprensión del transporte y de la movilidad en relación a la ciudad y el territorio, a partir de asimilar el binomio producción/atracción al de origen/ destino de viajes. Esto constituye una paradoja entre la teoría y la aplicación de dicho abordaje. Es preciso señalar que los binomios producción/atracción y origen/destino, no son lo mismo (Figura 2) ya que, mientras el primero focaliza en las componentes y características del hogar, como concepto explicativo de la movilidad, el segundo focaliza en los movimientos en el espacio, en los flujos y su impacto en las vías circulatorias, observando los efectos espaciales de los comportamientos sociales.

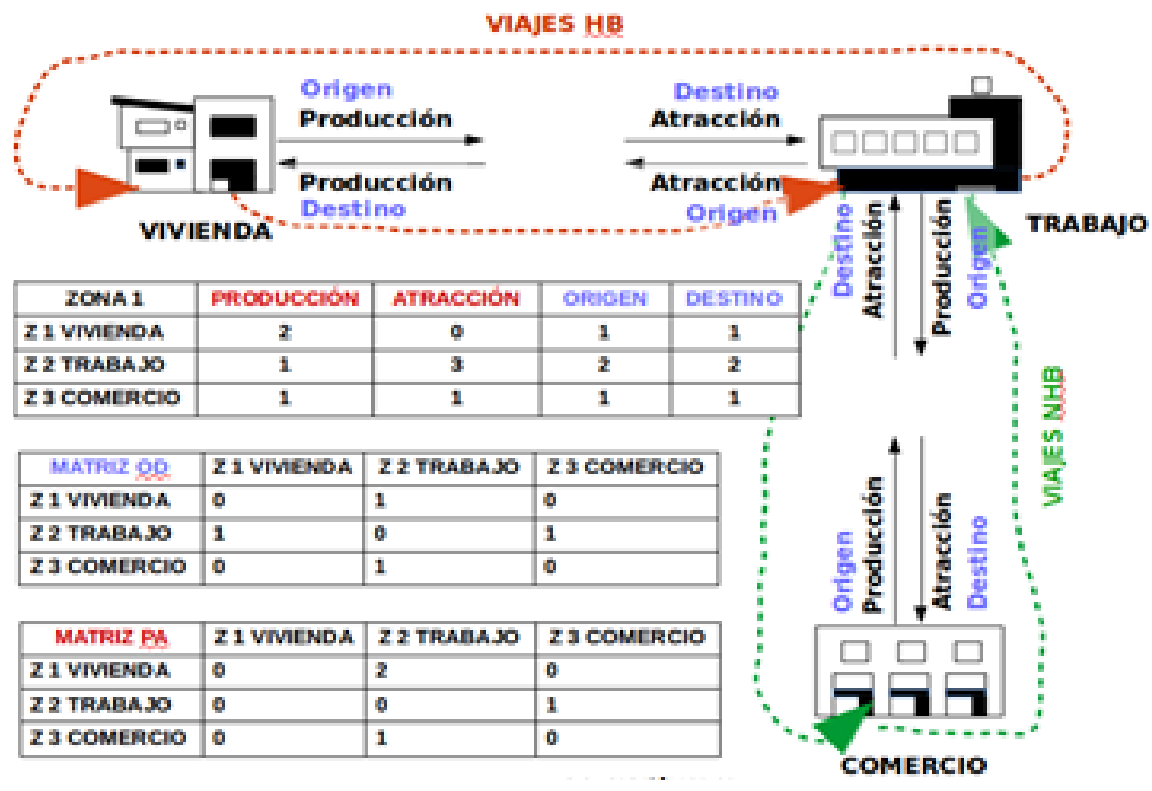

Figura 2. Esquema explicativo de las diferencias entre producción - atracción y origen -destino del viaje. Elaboración: Laura Aón. Fuente: Avances de tesis doctoral "Racionalidad no económica de la movilidad urbana de pasajeros".

Esta asimilación del binomio "producción/atracción" al de "origen/destino" es visible en el alcance habitual de los estudios basados en encuestas de origen y destino. Estos estudios, por un lado, persiguen el propósito de conocer la generación total de viajes de las ciudades, construyendo muestras en los lugares de la producción de viajes mediante encuestas a los hogares y sus miembros, a fin de poder construir matrices espaciales o espacializables de producción, atracción, generación, origen y destino de viajes (Figura 3). Sin embargo, los resultados finales y aplicados de estos estudios, suelen quedar reducidos a una espacialización de matrices de origen y destino que permiten dimensionar y analizar flujos de movilidad en las vías circulatorias, desvinculados de la información socio económica de los hogares que producen dichos flujos y reduciéndolos a un mapeo de los movimientos en el espacio de las calles o de los recorridos de transportes masivos (trenes, trolebuses, tranvías, colectivos, subterráneos). 

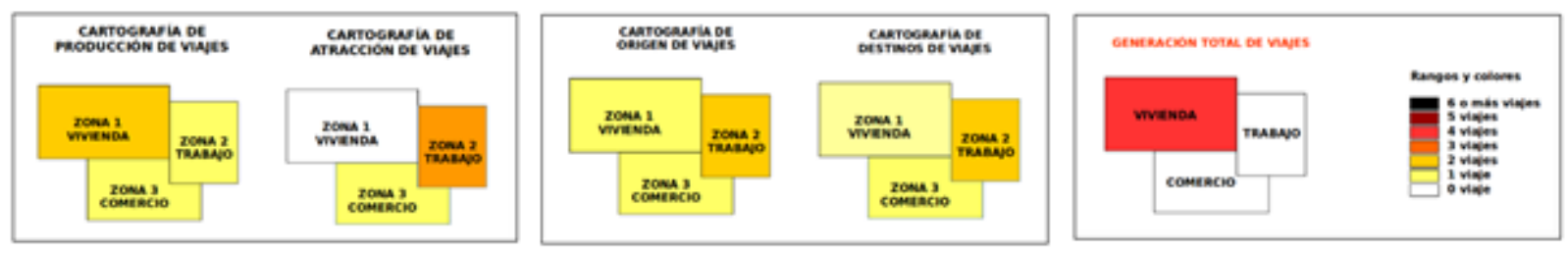

Figura 3. Mapeo de matrices de origen, destino, producción y atracción para un mismo caso de viajes. Elaboración: Laura Aón. Fuente: Avances de la tesis doctoral "Racionalidad no económica de la movilidad urbana de pasajeros".

A partir de estos resultados, las ciencias sociales tienden a equiparar los conceptos de producción/atracción de viajes a los de origen/destino de viajes en sus críticas a los estudios cuantitativos y espaciales de movilidad soslayando, o desconociendo, el peso específico de la teoría de la generación de viajes en la comprensión social y espacial de la movilidad. El basamento conceptual de la geografía del transporte, indica que el "transporte", como concepto, apela a un abordaje tecnicista, tecnócrata, lineal y abstracto, es decir, referido al abordaje de las infraestructuras y medios de transporte, exclusivamente (Gutiérrez, 2009). En este sentido, si por un lado la dimensión social y cultural implica mirar el problema en términos de las capacidades y necesidades de la población para moverse, en lugar de la capacidad de los medios de transporte para trasladar pasajeros de un punto a otro de un territorio dado (Miralles Guash y Cebollada, 2009); por otro lado tanto los estudios clásicos de transporte, como la teoría de la generación de viajes, incluyen relaciones socio-económicas y de género en sus análisis, dimensiones básicas para un abordaje sociocultural. Sin embargo, es cierto que la teoría clásica y sus mejores aplicaciones carecen de explicitaciones del tipo que encontramos en los estudios sociales de la movilidad. Es por ello que en este trabajo proponemos hacer un aporte en ese sentido.

\section{El atractor de viaje como fuente de información y análisis}

Los atractores de viaje constituyen una fuente de información clave para los estudios espaciales y cuantitativos de la movilidad urbana, estratégicos en dos dimensiones o aspectos. Una de ellas es su valor locacional y espacial único, ligado a sus funciones y oferta de actividades y servicios. La otra, es su entidad como parte del binomio de la generación de viajes, su relación con la producción de viajes. Estas características de los atractores los potencian como fuente de información, en la comparabilidad de las formas, condiciones y problemas de los viajes de diferentes individuos a ese mismo lugar y en la posibilidad de indagar a dichos individuos sobre condiciones clave de sus lugares de residencia donde se producen los viajes.

Entre las propuestas teóricas metodológicas de los modelos matemáticos de simulación de transporte, existen modelos que focalizan en destinos de viaje. Es el caso de los modelos gravitacionales y de los modelos Logit (Etcheñique, et al. 1975; De La Barra, 1989; Ortuzar y Willumsen, 1994; Lafuente, 2000). Los primeros son utilizados para abordajes más agregados y escalas interurbanas y los segundos se aplican en lugares de destino de viajes unitarios a partir del relevamiento de encuestas, haciendo foco en las variables espaciales cuantitativas ligadas al diseño de infraestructuras de circulación.

El relevamiento de encuestas en actividades no-residenciales, es decir actividades que implican importantes flujos de movilidad urbana, como la educación, el trabajo y la salud, constituye una estrategia de investigación relevante para estudios de escala urbana, especialmente si dicha escala permite reconocer atractores clave representativos de la matriz productiva de cada ciudad. En el caso particular de la ciudad de La Plata, 
una de sus principales actividades productivas está ligada a su rol como ciudad capitaladministrativa de la provincia de Buenos Aires. A su vez, La Plata se caracteriza por poseer una matriz productiva de carácter predominantemente terciario $(\mathrm{EPH}, 2019)$. En materia de educación, se trata de una ciudad universitaria, con una oferta destacada en cantidad de establecimientos de educación primaria y secundaria. Con respecto a su rol en materia de salud, constituye un atractor regional y provincial, con más de 10 centros de salud especializados, y de alta complejidad, para niños y adultos. A partir del relevamiento desarrollado en dichos atractores, se construye la información del viaje específico del individuo y de su hogar, dando cuenta de: i) las condicionantes espaciales (lugar de residencia); ii) materiales (nivel de ingreso, tasa de motorización y tamaño del hogar) y iii) sociales (género, nivel de instrucción, problemas y motivaciones específicas).

Para la realización de los diferentes estudios de movilidad en atractores, el camino metodológico estratégico se diseñó en cuatro etapas: las dos primeras relacionadas a la organización y gestión previa en campo, y las dos restantes relacionadas a la producción, procesamiento de datos y análisis de información. Los relevamientos en atractores se realizaron en diferentes etapas, según las actividades: salud, educación, trabajo. La selección de casos fue realizada a partir de criterios acordes a las búsquedas de la investigación del OMGLP, que siempre redunda en investigar los patrones de movilidad de pasajeros en la ciudad; esto incluye estudiar la criticidad de los viajes y analizar las diferencias de esa criticidad según grupos socio económico de la población, lugares de residencia, tamaño y estructura de los hogares involucrados. Basado en estas lógicas, la selección final de los atractores conformó una muestra representativa de los atractores más relevantes de la ciudad en tres aspectos: (i) grado de impacto: tamaño y capacidad de servicio que ofrecen; (ii) tipo de gestión de los establecimientos: públicos o privados y (iii) localización urbana: centrales, peri-centrales, peri-urbanas y periféricas.

\section{Encuestas de movilidad como proceso de aprendizaje colectivo}

El Observatorio de Movilidad del Gran La Plata (LII - IIPAC), con la finalidad de diseñar una estrategia metodológica incorporando las nuevas tecnologías para reconstruir la producción, atracción, generación, origen y destino de los viajes en las ciudades, comenzó a desplegar en el año 2013 y en base al marco teórico metodológico de la tesis doctoral "Racionalidad no económica de la movilidad urbana de pasajeros", un proceso continuo de aplicación, procesamiento y evaluación de encuestas (Aón et al., 2017). Dicho proceso consolidó la construcción de una propuesta de análisis de la movilidad en base a la combinación de nuevas tecnologías (encuesta plataforma web y georreferenciación en sistemas de información geográfica), tomando como fuente de información y unidad de análisis, a los denominados "atractores de viaje".

Para poder entender el trabajo de relevamiento como un proceso continuo, resulta clave visibilizar las estrategias implementadas en los reajustes, en busca de ganar efectividad y simpleza, tanto en los procesos de recolección y carga de los datos, como en su procesamiento y en los resultados. En este proceso continuo se distinguen dos momentos o etapas de diseño, aplicación, revisión y procesamiento (Figura 4): un primer momento de encuestas de producción de viajes no-domiciliarias, y un segundo momento de encuestas de atracción de viajes.

El primer momento se inicia con el proceso de adaptación de las encuestas de movilidad tradicionales a un formato web autoadministrado. Se exploraron diversas plataformas web aptas para encuestas de movilidad y se diseñaron nuevos formularios de encuesta, basados en una crítica a la estructura de la encuesta domiciliaria tradicional. Las partes tradicionales de la encuesta domiciliaria de transporte incluyen formularios 
de vivienda, hogar, personas y viajes, mientras que las partes de la nueva encuesta de movilidad (ya no domiciliaria) incluirían formularios de vivienda, hogar, persona y actividades (principales y secundarias) como nueva categoría de relevamiento y análisis de la movilidad. Esta nueva categoría fue incluida con el propósito de relevar los principales atractores de viaje de las personas.

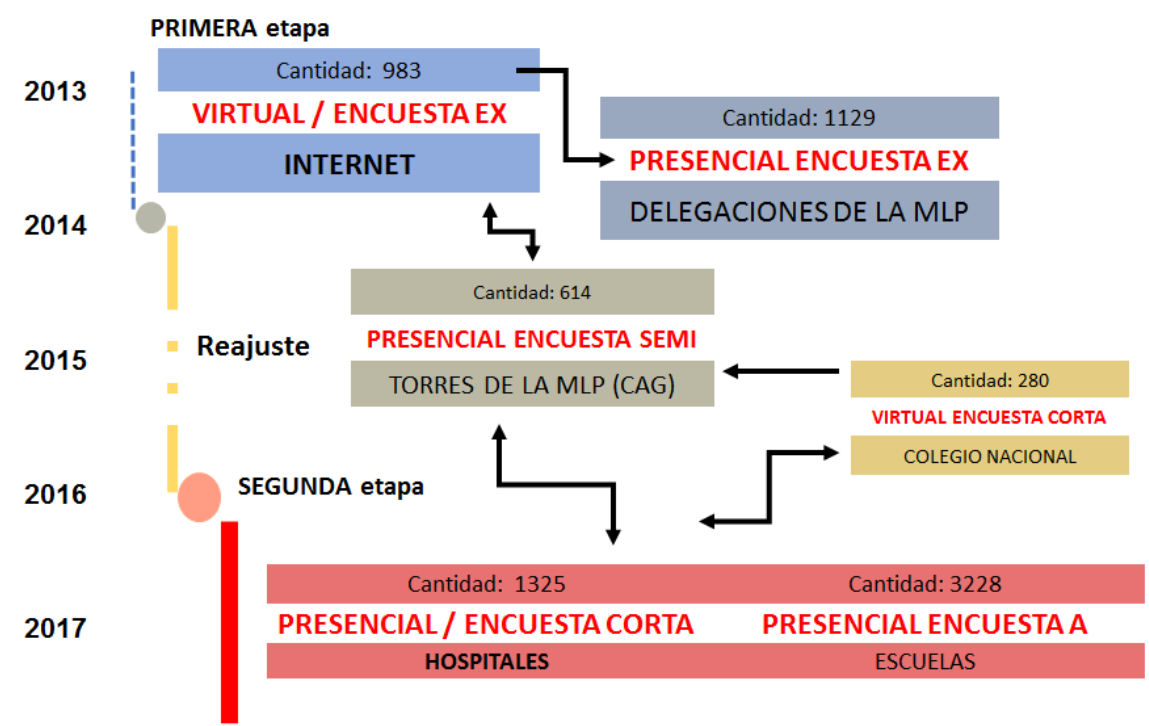

Figura 4. Etapas de relevamiento y procesamiento de las encuestas de movilidad. Elaboración: Dr. Arq. María Julieta López.

El segundo momento es donde, a partir de reflexionar sobre el procesamiento y los resultados del primero, se diseñan y aplican encuestas en atractores de viaje. En esta segunda etapa todas las encuestas fueron presenciales y se realizaron en tres tipos diferentes de atractores: i) centro administrativo del trabajo gubernamental; ii) establecimientos de salud privados y públicos y iii) establecimientos educativos primarios y secundarios. A su vez, en cada una de las experiencias se pueden distinguir tres momentos metodológicos del relevamiento: i) un momento previo de logística y organización, ii) un momento de ejecución del trabajo de campo y iii) un momento posterior de posproducción y procesamiento.

\section{Primera etapa: la encuesta web y el relevamiento en delegaciones municipales}

En el momento en que se lanzó la encuesta de movilidad urbana en el año 2013 (Aón, 2013), el Observatorio de Movilidad para el Gran La Plata (OMGLP) funcionaba de manera inicial como una plataforma web. Su principal objetivo era avanzar en el desarrollo metodológico combinado de técnicas cuantitativas y cualitativas, que permitiera incorporar progresivamente nuevas tecnologías, evaluando en paralelo alternativas de herramientas de relevamiento mediante "pruebas piloto" y, a la vez, evaluando los sucesivos datos obtenidos. La puesta en marcha del OMGLP como plataforma de construcción y exposición de información, se origina en este primer momento.

En la primera etapa, se trabajó con encuestas web enviadas a través de correos electrónicos y redes sociales y luego, en una subetapa se realizaron encuestas presenciales en las delegaciones y centros comunales del partido de La Plata, con el objetivo de completar una muestra estadísticamente representativa de la periferia del partido y de los tipos de hogar por barrio. El propósito ulterior era que los resultados pudieran ser expandidos al 
conjunto de la población de la ciudad y pudieran ser adaptados para calibrar un modelo integrado de usos del suelo y transporte (Aón et al., 2017). En un principio se hicieron 983 encuestas web y se completó con 1.129 encuestas presenciales en las delegaciones de la periferia de la ciudad. Se recabaron datos sobre 7.500 personas, aproximadamente, entre los viajes de los encuestados y de los miembros de sus hogares, el equivalente al $1 \%$ del universo poblacional (650.000 habitantes) del área de estudio al año 2010 (INDEC, 2010).

Como explica Aón (2017), la encuesta web es una herramienta innovadora y adecuada para conocer rápidamente los patrones de motivos y modos de viaje de una ciudad "intermedia" (hasta 700.000 habitantes aprox.). Además, resulta valiosa para capturar opiniones de los usuarios de los distintos sistemas de transporte. Dado su sistema de gestión autoadministrado, permite producir resultados a corto plazo con carga automática e informes estándar de la plataforma web contratada a tal efecto. Cabe destacar que, en comparación con las encuestas domiciliarias de origen y destino de viajes, son encuestas que recaban menor detalle de información, tienen procedimientos más sencillos de carga y, por lo tanto, resultan de mucho menor costo.

\section{Tareas Previas}

En primer lugar, se procedió a la etapa de diseño del formulario de las encuestas domiciliarias y su adaptación a plataforma web, con sus respectivas pruebas-piloto. Una vez lanzada la encuesta se realizó una fuerte difusión mediante redes sociales y la plataforma de la UNLP (Figura 5). Para posibilitar el trabajo de campo presencial de las delegaciones municipales, hubo un trabajo previo de gestión y coordinación, enmarcado en el convenio de la UNLP con el municipio de La Plata (MLP). Esto incluyó el contacto y la coordinación de horarios y días de relevamiento con los administrativos encargados de las diferentes delegaciones y la coordinación con choferes del municipio para el traslado del equipo de encuestadores a sus respectivos puntos de encuestas.

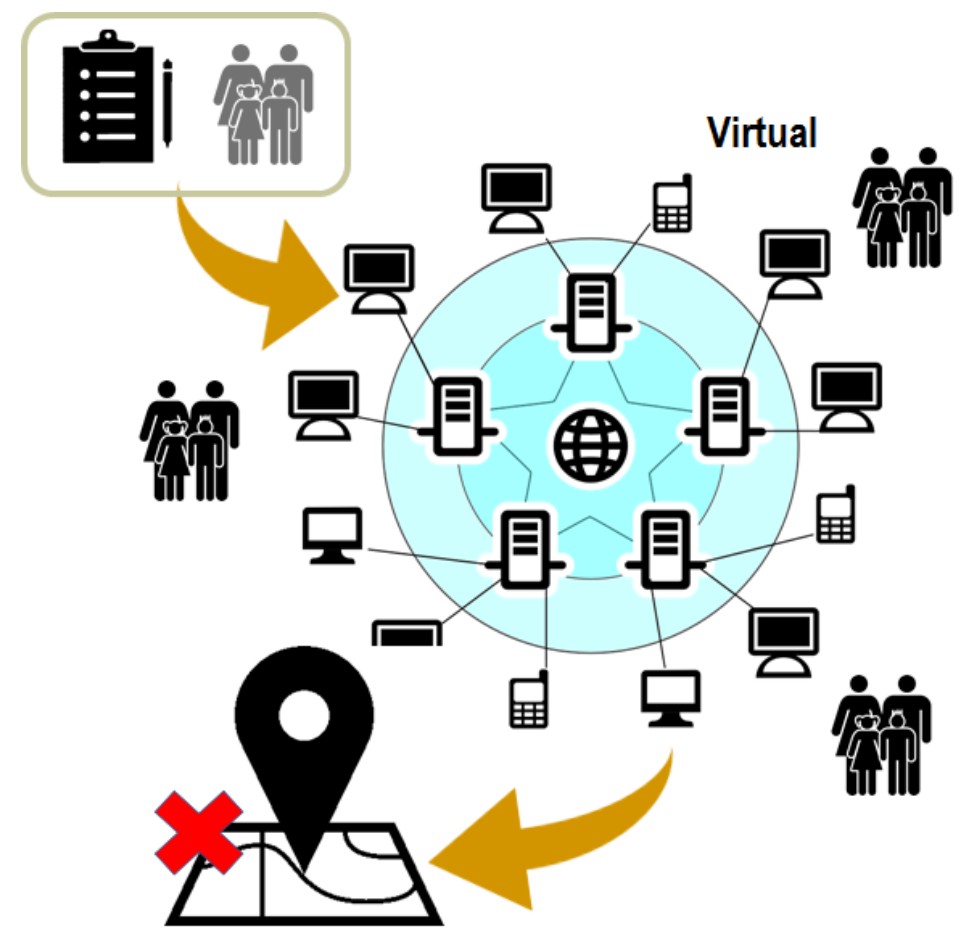

Figura 5. Proceso de relevamiento de encuestas de movilidad por medio de plataforma web. Elaboración: Dr. Arq. María Luciana Giglio. 


\section{Trabajo de campo}

Las encuestas autoadministradas vía-web requieren un grupo reducido de personal calificado: dos personas para el diseño del formulario y del operativo de recolección de los datos y dos personas para la planificación y gestión de contactos, grupos clave y estratégicos en redes sociales e internet. La recolección presencial de encuestas mediante encuestadores fue un operativo complementario diseñado en base a los resultados incompletos de la encuesta web. Se seleccionaron las delegaciones municipales periféricas que habían quedado poco representadas en los resultados iniciales y se diseñó un operativo de campo para el completamiento. Se trabajó con el mismo formulario y se capturó información de la persona y de quienes componían su hogar (Aón, 2017).

Para el relevamiento presencial fueron necesarios diez encuestadores, trabajando tres horas por día durante dos semanas y dos supervisores en campo. Las jornadas de relevamiento se realizaron los primeros días hábiles del mes ( 1 al 10) que coincidían con el pago de servicios ${ }^{1}$, el horario era 10 a 13 hs. Se distribuían las encuestas con lapiceras a lo largo de las filas de espera para trámites y pagos y se asistía con las dudas que pudieran surgir (Figura 6). El número de encuestadores y supervisores fue acorde a la estrategia de relevamiento que se diseñó, conforme a los resultados parciales de la muestra construida vía web. Es importante destacar que la experiencia presencial de encuestas permitió registrar, como metadato, las dificultades de comprensión del cuestionario por parte de los encuestados, y la importancia de la asistencia del encuestador para lograr obtener las respuestas deseadas.

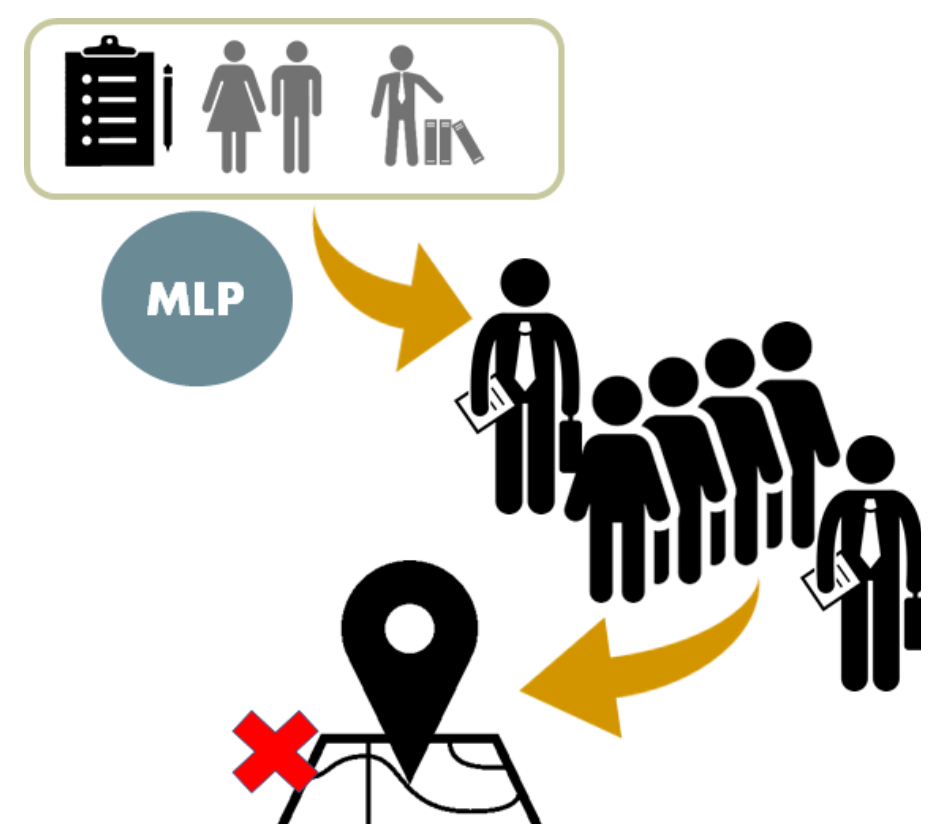

Figura 6 Proceso de expansión de encuestas de movilidad en delegaciones de la periferia del Partido de La Plata. Elaboración: Dr. Arq. María Luciana Giglio.

\section{Tareas de Posproducción y procesamiento}

El trabajo posterior de procesamiento de la encuesta requirió tres personas en laboratorio para verificación y análisis. Dicho trabajo insumió un año y dos meses hasta obtener resultados válidos por barrio, con detalle de patrones modales y de motivos de 
viaje, tasas de generación y matrices de producción y atracción de viajes (Aón, 2017). Las tareas de procesamiento de los resultados incluían: carga de los datos y georreferenciación de los encuestados de forma manual.

Esta última tarea particularmente, la georreferenciación de los encuestados, resultó dificultosa por falta de datos o información mal anotada por los encuestados o encuestadores. Esto repercutió en la pérdida de registros. La experiencia de aplicación de las encuestas autoadministradas puso en evidencia que el dato del lugar de residencia de los encuestados, que constituye un dato clave del estudio de patrones espaciales, había sido el dato donde más errores se registraban y el más difícil de capturar con certeza, sin la intervención del encuestador.

Por otro lado, pudo observarse que, en la etapa de encuestas autoadministradas online, los encuestados tendían a explayarse en las opciones de respuestas abiertas, lo cual puso en evidencia que era factible obtener respuestas en cuestionarios relativamente extensos, sin presencia de encuestador. Esta experiencia de campo dio cuenta de la necesidad de compactar el cuestionario en las encuestas presenciales, donde la gente se encuentra "de paso". En este sentido, se diseñó un nuevo formulario que permitió reducir el contenido de la encuesta a la información mínima necesaria para trabajar a nivel de hogares y a nivel de ciudad a la vez.

Finalmente, se observó que, en la etapa de encuestas autoadministradas, los encuestados respondiendo desde sus computadoras tendían a explayarse en las opciones de respuestas abiertas, generando información cualitativa valiosa en relación a la calidad de viaje y según su modo de transporte. Este resultado demostró el potencial de las encuestas web como método de recolección cuando se brindan las condiciones de confort que permiten el no estar "de paso" en el espacio público, sino en la comodidad de su casa. Esta situación se fortalece aún más al formular preguntas sobre las cuales el encuestado quiere expresarse y tiene opiniones para explayarse, como el estado de las infraestructuras y la calidad del transporte público, entre otros.

\section{Reajuste metodológico: un cambio de estrategia}

Luego de la primera etapa se dio un cambio importante en la estrategia, no solo del abordaje conceptual sino también desde el operacional, con la forma de implementación de la encuesta. A partir del trabajo y los resultados obtenidos de las encuestas presenciales en las delegaciones municipales, habiendo completado la información sobre patrones de movilidad a nivel de delegación para la ciudad de La Plata, se comienza a revisar y consolidar el diseño de la encuesta orientada a los atractores de viaje.

La primera versión de formulario de encuesta en atractor de viaje fue desarrollada y probada como encuesta en el Colegio Nacional Rafael Hernández, en el marco de un proyecto de extensión universitaria desarrollado desde el Observatorio de movilidad ${ }^{2}$. Se diseñaron cuestionarios breves, buscando capturar datos de producción (residencia, hogar, miembros, trabajo, motorización) y atracción de viajes (motivo, modo, hora, costo) en el mismo cuestionario.

El nuevo diseño de formulario de la encuesta tuvo como propósito tomar información del viaje al atractor y, a la vez, recuperar información sobre el hogar de los encuestados. Por tanto, el análisis desde los atractores de viaje constituía una suerte de «camino 
heurístico» para el estudio de la movilidad, como práctica ligada a las características socio-económicas de los hogares y a las características espaciales del lugar de residencia en la ciudad (Aón, 2017).

Para esta nueva etapa se capitalizaron los aprendizajes de la experiencia de aplicación de las encuestas autoadministradas y del proceso de adaptación presencial, a partir de lo cual se buscó simplificar las preguntas, la forma de registro y las opciones al encuestado. La compactación del cuestionario apuntó a la celeridad de las respuestas, dando cuenta de la situación "de paso" o de apuro de los encuestados en los atractores donde se los intercepta.

La incorporación de encuestadores en las delegaciones municipales permitió, a partir del relato de sus experiencias, compactar el cuestionario, permitiendo reducir "las redundancias" que se habían diseñado como medidas de verificación para los cuestionarios autoadministrados, pero que ya no lo requería un formato de cuestionario presencial. También se revisó el procedimiento de carga de las encuestas en el soporte web, y se construyó un documento instructivo para futuros relevamientos con una guía de pautas para la georreferenciación de las encuestas en Google maps, compatible con sistemas de información geográfica estándar, que ayudó a garantizar el uso de la totalidad de los registros realizados.

\section{Segunda etapa: los atractores de viaje}

Esta etapa se caracterizó por desarrollar la metodología de relevamiento en atractores de viaje. Inicialmente, se definieron los tipos de atractores a relevar en concordancia con la matriz productiva de la ciudad. En el caso de La Plata, la administración pública, salud y la educación son actividades clave, por lo que se seleccionó para la segunda etapa de las encuestas, tres tipos de atractores, relevados en el siguiente orden: i) centro administrativo del trabajo gubernamental; ii) establecimientos de salud privados y públicos y iii) establecimientos educativos primarios y secundarios.

Este tipo de encuesta por atractor requiere, al igual que las encuestas domiciliarias o en productores de viaje, un trabajo previo para la definición del tamaño de la muestra de atractores. Dicha muestra se determinó según el tipo de atractor, la actividad que se desarrollaba en el mismo, el tamaño, el tipo de gestión (en el caso de salud y educación), la localización territorial y los horarios de atención al público. Los relevamientos se realizaron en diferentes tiempos, acordes al tipo de atractor a relevar, y se desarrollaron en el siguiente orden cronológico: en primer lugar, los atractores de la administración pública de La Plata, luego el relevamiento de atractores de salud y, finalmente, el relevamiento de los atractores por educación.

Asimismo, al igual que las encuestas domiciliarias, las encuestas en atractores requieren una coordinación administrativa y logística con instituciones, tanto públicas como privadas, para lo cual se desplegaron nuevas estrategias. En el primer caso, la coordinación fue con las autoridades del municipio, mientras que, en el caso de los hospitales y de las escuelas la coordinación fue con las autoridades de cada uno de los establecimientos. Este trabajo inicial de coordinación administrativa implicó concretar (a) reuniones con el cuerpo directivo de cada institución para explicar el operativo, (2) conseguir las autorizaciones internas para la realización del trabajo, (3) gestionar las autorizaciones para difundir internamente el operativo de campo a fin de informar previamente a las personas a ser encuestadas, (4) conseguir autorizaciones individuales para el ingreso de encuestadores a cada institución con las autoridades gubernamentales pertinentes, (5) acordar y definir las fechas y los horarios para la realización del trabajo de campo. 
En esta etapa se definió con mayor detalle, como revisión de la etapa anterior, el procedimiento en al momento del volcado de los formularios impresos por parte de los encuestadores, a partir de definir un sistema de referenciación previo para cada encuesta. Para cada registro, es decir las respuestas correspondientes a cada encuestado, se asignó un número de identificación o ID que permitiera vincular esta información con su localización residencial estimada a partir del uso de sistemas de información georreferenciada.

El operativo de campo se concretó con encuestadores agrupados de a dos en distintos turnos: Mañana / tarde / noche, según el caso. La actividad de cada encuestador estaba compuesta de dos momentos. Por un lado, el levantamiento de la encuesta en el atractor y por otro, el volcado del formulario impreso en la plataforma web y la georreferenciación de cada encuesta. El levantamiento en atractores utilizó mayormente formularios impresos. Donde se pudo, se utilizó wi-fi y las encuestas fueron autogestionadas, pero con asistencia de los encuestadores que estaban presentes.

La carga de datos se realizó diariamente en la plataforma web contratada y mediante el sistema de referencia ID se relacionaba con la georreferenciación de los lugares de residencia de los encuestados, que también se cargaban diariamente. El procesamiento fue realizado por cada encuestador, la vinculación y combinación de todos los datos quedaban a cargo de un coordinador (Aón, 2017). A continuación, se detallan en mayor profundidad las experiencias que se realizaron en cada tipo de atractor.

\section{Atractores por trabajo: el caso del Centro Administrativo Gubernamental}

Los atractores que se relevaron inicialmente, fueron una selección de atractores de la administración pública municipal y provincial, correspondientes a las torres administrativas de gobierno. En ellas se aplicó el formulario de la encuesta web adaptada, focalizando en la página de actividades. Para definir el tamaño de la muestra a encuestar, en este caso, se dimensionó el total de gente que asiste al lugar, las diferentes franjas horarias de actividad y los diferentes perfiles ocupacionales y niveles socio-económicos. En las torres de gobierno se realizaron 614 encuestas a los administrativos y técnicos. Este operativo requirió la coordinación con las autoridades de la MLP para definir días de relevamiento en las torres.

El periodo de relevamiento fue de dos semanas y el formulario aplicado, como ya se mencionó, fue adaptado con respecto a la versión web original. El ajuste tuvo como criterio la posibilidad de integración, compatibilidad y comparabilidad de resultados con las encuestadas realizadas en etapas previas. En lo operativo, los formularios de encuestas se distribuían en las oficinas a partir de media mañana, hasta que se agotaban las encuestas en papel y se retiraban las encuestas al día siguiente, o cuando así lo indicaran las autoridades de la oficina. Inicialmente los encuestados esperaban porque los empleados completaran la encuesta, pero los ritmos de su trabajo no permitían en muchos casos las interrupciones (Figura 7). 


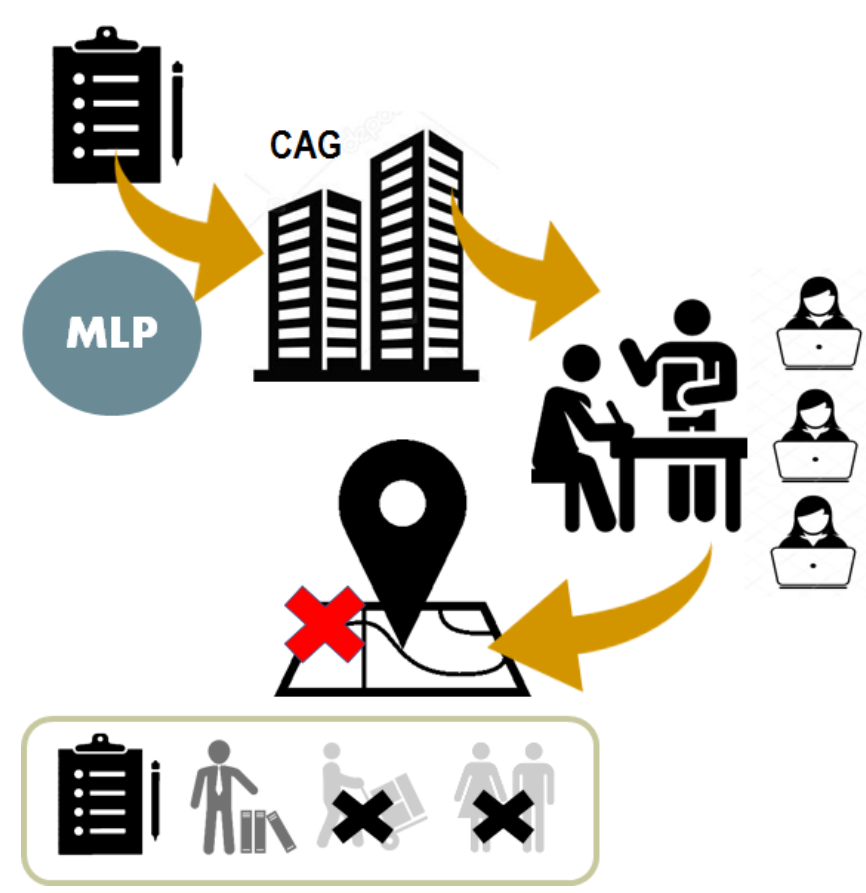

Figura 7. Proceso de expansión de encuestas de movilidad en un atractor laboral característico del Partido de La Plata. Elaboración: Dr. Arq. María Luciana Giglio.

El procesamiento de la encuesta incluía la carga manual a la plataforma web, a cargo de algunos encuestadores. En este proceso se presentaron dificultades en la etapa de georreferenciación de los domicilios de los encuestados, por falta de datos o información mal anotada. Algunos datos de domicilio fueron inferidos por otros datos de la encuesta que daban cuenta de los lugares más frecuentados por el encuestado.

Este relevamiento fue parte del completamiento de la muestra de movilidad del Gran la Plata, alcanzando las 2000 encuestas con representatividad territorial estadística a nivel de localidad. Esto fue posible gracias a la construcción de una nueva expansión de la muestra obtenida, utilizando datos de población provistos por el censo de Población 2010 proyectados a 2014 (INDEC, 2010) El modelo de expansión incluyó la agregación de hogares por tamaño y por localidad. De esta manera se llegó a consolidar la información de patrones modales de movilidad 2013-2014, para el Partido de La Plata (Aón, Giglio y Cola, 2016).

Luego se inicia una etapa de conversaciones respecto al interés del Observatorio de Movilidad, acerca del nivel de accesibilidad de los habitantes de la ciudad de La Plata a los equipamientos de Salud y Educación del Gran La Plata, en el marco del trabajo colaborativo entre el L2 IIPAC y la Dirección de Transporte y Tránsito de la Municipalidad de La Plata. Por un lado, la accesibilidad del transporte público a estos equipamientos era un tema de quejas de los usuarios y, por lo tanto, estaba en la agenda local. Por otro lado, la salud y la educación habían sido dos líneas de investigación histórica que los investigadores pretendían recuperar y poner en valor. En este contexto, surge la idea de avanzar conjuntamente con el estudio de atractores de viaje en hospitales y centros de salud del Partido de La Plata.

\section{Atractores De Viajes Por Salud}

El trabajo de encuestas en atractores de viajes de salud, realizado entre julio y agosto del año 2016, fue financiado por el sindicato de la UTA La Plata (Unión Tranviarios 
Automotor). El trabajo comienza con la presentación al secretario general de la Uta La Plata, de una propuesta metodológica para un relevamiento que incluyó la selección de centros de salud a relevar y el diseño de un formulario de encuesta a aplicar en cada caso.

Este formulario desarrollado tenía dos formatos, uno corto y otro extendido. El corto estaba orientado al registro de la atracción de viaje propiamente dicha, es decir, el registro solo del viaje del encuestado al atractor, con información cuanti y cuali de ese viaje y de su lugar de origen. El extendido, estaba orientado a completar el registro de la información de la producción del viaje del encuestado y de los miembros del hogar. El formulario extendido tenía el propósito a su vez, de producir mayor información de movilidad para ser integrada a la muestra de movilidad precedente 2013 - 2014.

Respecto del formulario original de la encuesta de movilidad, y sus sucesivas adaptaciones, éste nuevo formulario presentado, se caracterizó por ser específico para distinguir el tipo de atractor a relevar y el rol del encuestado o bien la actividad que pudiera desempeñar en el atractor de viajes por salud (paciente, enfermero, acompañante, etc.). Además, se incorporó el número de ID para facilitar la posterior georreferenciación.

La coordinación previa con las autoridades de los establecimientos de salud fue una etapa clave, ya que se trató de un trabajo de gestión intenso, de 45 días previos al inicio de los trabajos de campo, para el que se debieron construir agendas de directores y personal responsable de cada establecimiento seleccionado, pautar reuniones en cada caso y preparar material para la explicación del trabajo, objetivos y operativo. Este procedimiento se realizó con 30 establecimientos de salud, aunque no todos resultaron autorizados.

La capacitación del equipo de encuestadores fue otro de los aspectos clave del trabajo. Se diseñó un instructivo y un operativo de completamiento de encuestas y carga de la información en la plataforma web, a cargo de la misma persona, tanto de los datos de movilidad recabados como de la georreferenciación de los lugares de residencia de las personas encuestadas en google maps, a partir del ID asignado a cada encuesta. Se instruyó además a los encuestadores a cargar las encuestas en el mismo día de la realización del relevamiento, de modo de poder saldar cualquier duda a partir de la memoria de corto plazo.

Los criterios de selección de los establecimientos fueron: el grado de influencia del establecimiento (local, zonal, regional), el tipo de gestión (pública o privada) y su localización (periférica o central). Para obtener una muestra completa de la situación, se diseñó un operativo en jornadas de 3 horas en turno mañana y turno tarde, en coincidencia con los principales horarios de atención en consultorio. El periodo de relevamiento elegido también fue parte de las decisiones del operativo de campo, que se desplegó en la etapa del año de mayor demanda de actividad sanitaria en la ciudad, que es el periodo invernal: cuatro semanas de invierno, las dos últimas de julio y las dos primeras de agosto.

Los espacios seleccionados en cada establecimiento fueron las salas de espera, donde se colocaron previamente carteles informativos acerca del operativo de encuestas. Los días y turnos acordados, los encuestadores en grupos de dos asistían a las salas de espera de las guardias y consultorios, preguntaban a las personas si querían participar de la encuesta y, como incentivo para realizar la encuesta larga, se ofrecía participar de un sorteo por carga en la tarjeta SUBE (Figura 8). Paralelamente, se les entregaban formularios de encuestas para llenar al jefe de personal del establecimiento de salud, con la consigna de que sean repartidas entre el personal, a fin de obtener registros de los empleados del establecimiento. 

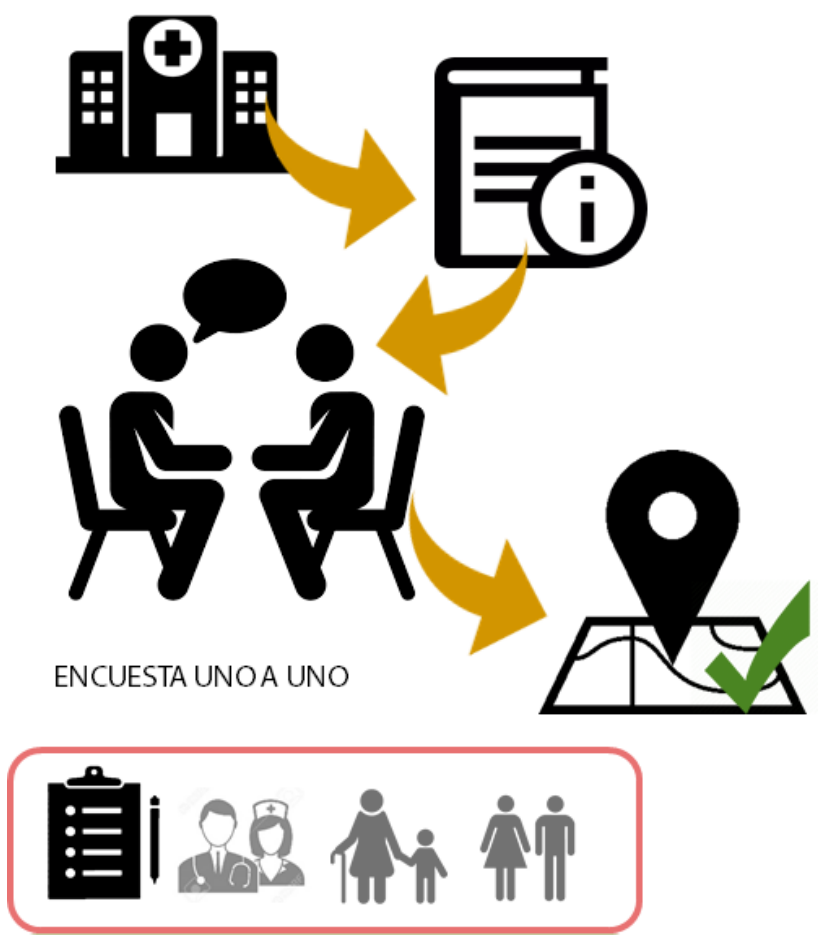

Figura 8 Proceso de expansión de encuestas de movilidad en establecimientos de salud. Elaboración: Dr. Arq. María Luciana Giglio.

Finalmente, el procesamiento de los datos fue realizado acorde a las pautas iniciales, realizando cada encuestador la carga manual de sus encuestas en la plataforma web, así como también elaborando la georreferenciación de los lugares de residencia de sus encuestados, en google maps a partir del ID del encuestado. El resultado fue la totalidad de los registros (1.325) recuperados y completos.

\section{Atractores De Viajes Escolares}

El trabajo de encuestas en atractores de viajes de educación, realizado en noviembre del año 2016, fue también financiado por el sindicato de la UTA La Plata (Unión Tranviarios Automotor). El trabajo comienza con el cierre del estudio de atractores de viajes de salud, con la presentación al secretario general de Uta La Plata, de la metodología de trabajo, la selección de escuelas y del diseño del formulario de encuesta a realizar.

El formulario desarrollado para los atractores de educación fue similar al desarrollado para los atractores de viajes por salud, con un formato corto solamente. El ajuste que se realizó implicó el orden cómodo de respuesta por parte de los encuestados, focalizando el inicio de las preguntas en las formas, modos y tiempos de viaje al atractor, articulando luego la información de su lugar de residencia y características del hogar y evitando la desarticulación de preguntas relativas a información personal sensible, tal como el horario en que no está en su casa u otra información de familia y vivienda.

El trabajo de coordinación previa con las autoridades de los establecimientos educativos requirió en este caso de la gestión de una autorización del Ministerio de educación de la provincia de Buenos Aires, para luego poder coordinar con las autoridades de los establecimientos educativos provinciales seleccionados. En relación a los establecimientos educativos dependientes de la Universidad Nacional de La Plata, solamente fue necesario un aval universitario como Observatorio de Movilidad. 
La capacitación del grupo encuestador, en este caso, incluyó ajustes orientados a la forma de recoger los datos en la situación áulica y el apoyo en elementos gráficos para apuntar la fluidez de respuesta, en el caso de las escuelas primarias y de niños más pequeños. Se aplicó el mismo instructivo de carga que en la experiencia anterior.

Los criterios de selección de los establecimientos educativos fueron: (1) el tamaño de la matrícula escolar, (2) la localización (periférica o central) y (3) su tipo de gestión (pública o privada). Para obtener una muestra completa de la situación de cada establecimiento educativo, se diseñó un operativo en jornadas de 3 horas en turno mañana, turno tarde y turno vespertino, en coincidencia con los horarios de clase de cada escuela. El periodo de relevamiento fue de cuatro semanas entre octubre y noviembre, considerando que el periodo lectivo en la ciudad de La Plata y en estas escuelas culmina entre mediados del mes de noviembre y mediados del mes de diciembre.

El trabajo de campo consistió en jornadas de 3 horas, turno mañana y turno tarde. Los espacios seleccionados en cada establecimiento fueron las aulas. El relevamiento consistía en el arribo de los encuestadores, en grupos de a dos, a cada aula, junto a alguna autoridad del establecimiento. Se preseleccionaron cursos que fuesen, en lo posible, de edades diferentes, a fin de capturar la diversidad de formas de movilidad en relación a grupos etarios. Una vez en el aula, se presentaba la encuesta, se distribuían los formularios y se organizaba su completamiento en forma conjunta, con todos los alumnos presentes, incluido el docente o preceptor a cargo del curso, bajo las instrucciones de los encuestadores presentes (Figura 9).

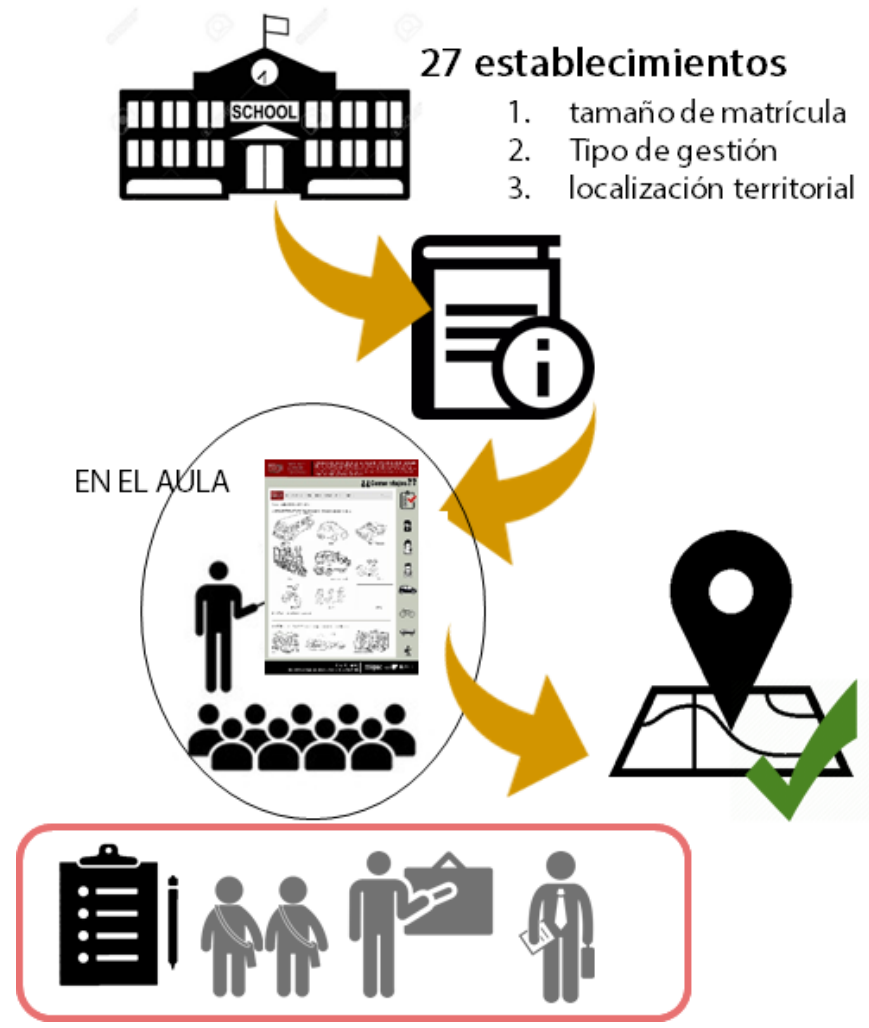

Figura 9 Proceso de expansión de encuestas de movilidad en establecimientos de salud. Elaboración: Dr. Arq. María Luciana Giglio.

El procesamiento de los datos obtenidos fue realizado acorde a las pautas iniciales presentadas en la etapa de capacitación. Cada encuestador fue responsable de la carga manual de sus encuestas en la plataforma web, así como también de su correspondiente 
georreferenciación en google maps a partir del ID del encuestado. Los encuestadores realizaron el trabajo de carga y georreferenciación en el día del trabajo de campo y, así como en el caso de los establecimientos de salud, este sistema de relevamiento garantizo la totalidad de los registros (3.228) georreferenciados y completados.

\section{Conclusiones}

Las principales conclusiones se desarrollan en torno a lo metodológico, exponiendo dos binomios analíticos de juicio a los instrumentos aplicados: encuesta de producción de viajes - encuesta de atracción de viajes, y encuesta web - encuesta presencial. Señalando, a su vez, la importancia de poner en valor aspectos de la teoría del transporte, reconociendo el valor del estudio de atractores en sí mismo y para el abordaje integral de la movilidad y destacando el valor del trabajo en equipo y de la construcción colectiva de conocimiento en la operacionalización de los trabajos de campo.

\section{Sobre los instrumentos aplicados}

La encuesta web es una de las herramientas estructurales en el proceso de construcción de información primaria del Observatorio de Movilidad del Gran La Plata, aunque no sea la única. A pesar de que la plataforma web contratada resultó óptima en términos cuantitativos, económicos y temporales, el mayor desafío para su aplicación fue, y continúa siendo, el diseño del formulario de encuesta, dada la complejidad del tema que se investiga. Presentamos dos binomios analíticos para juzgar el instrumento: encuestas de producción - encuestas de atracción; encuestas virtuales - encuestas presenciales.

Para el primero, es preciso distinguir las dificultades y complejidad que requiere cada tipo de encuesta (de producción y de atracción) en relación a los propósitos de cada una. Las de la primera etapa, focalizadas en la producción de viajes, estuvieron centradas en conseguir, en un corto plazo, la construcción de la información de movilidad necesaria para conocer los patrones de movilidad, por localidad, de la totalidad del Partido de La Plata. Información que, tradicionalmente, se consigue con encuestas domiciliarias de altísimo costo y que en la ciudad de La Plata no se realiza desde el año 1993. Las encuestas de la segunda etapa, focalizadas en la atracción de viajes, estuvieron centradas en conseguir en un corto plazo, la construcción de información de movilidad necesaria para conocer los patrones de movilidad del atractor analizado; con un objetivo potencial de mediano plazo de relevar la totalidad de los atractores de viajes representativos de la matriz productiva de La Plata, para entonces tener los patrones de movilidad de la ciudad.

En esta contrastación se justifica el proceso de simplificación de formularios entre una etapa y otra. Es decir, no se trata de que un tipo de encuesta pueda reemplazar a la otra, sino que toman caminos diferentes para llegar a un mismo tipo de resultado, los patrones de movilidad a nivel ciudad. Las encuestas de la primera etapa permitieron lograr el objetivo de conocer estos patrones, mientras que las encuestas de la segunda etapa, lo han hecho parcialmente. Queda un camino por recorrer, que permita registrar patrones de movilidad en atractores universitarios, industriales, en la construcción, el empleo doméstico, el sistema judicial y el servicio inmobiliario, por mencionar los atractores clave de la matriz productiva de esta ciudad (EPH, 2019).

En cuanto al binomio analítico de encuestas web - encuestas presenciales, existe un factor de costos de recursos económicos y de tiempos, asociado a la elección de dicho sistema, el cual es crítico al momento de utilizar una u otra forma de relevamiento. Este factor de costos, ha generado el desfasaje temporal de los relevamientos, así como 
también ha requerido cambios en los formularios, acordes al tipo de encuesta que fuese posible hacer. Generando de esta forma algunos problemas de incompatibilidad en el procesamiento de encuestas web y presenciales y, a la vez, fomentando un aprendizaje para las siguientes experiencias en las cuales se diseñaron formularios con mayor flexibilidad.

En el caso de las encuestas virtuales, las cuales carecen de encuestador o guía, precisan de una elaboración de formularios muy simplificados para el encuestado. Es decir, de fácil interpretación, comprensión y respuesta, a la vez que requieren generar cierta redundancia de preguntas para la verificación de información clave de la encuesta, previendo errores de interpretaciones u omisiones. Como contracara de la simplificación de los formularios para el encuestado, se asume una mayor complejidad en los procedimientos para el procesamiento de los resultados obtenidos. En el caso de las encuestas presenciales, necesitan de una menor complejidad, tanto del diseño del formulario como del procesamiento posterior, pero compensan esto al poder prescindir de estrategias de redundancia que garanticen la validez de los resultados, como en el caso de las encuestas autoadministradas. Esto es posible gracias a la valiosa asistencia del encuestador presente frente al encuestado, con lo cual se corrigen o eviten errores en la interpretación de las preguntas, se captan opiniones y explicaciones sobre la conducta de los encuestados y, por último, se garantiza la calidad y completamiento de los registros.

\section{Sobre los costos y tiempos involucrados}

El factor "costo" ha resultado una clave para el desarrollo de éstos nuevos instrumentos del Observatorio de movilidad y fue el motivador principal de la exploración de nuevas tecnologías para la construcción de información para el Gran La Plata. Esta ciudad no contaba con datos de transporte y movilidad desde 1993, fecha de la anterior encuesta de transporte, realizada mediante la clásica metodología de muestreo domiciliario. Durante los años 2008 a 2013, el Banco Mundial, a través del Ministerio de Economía primero, y a través del Ministerio del Interior después, financió mediante el programa PTUMA Y PTUBA, estudios de origen y destino en ciudades grandes e intermedias de nuestro país. Desde el Observatorio, se procuró acceder a estos financiamientos, pero no se consiguieron por falta de respaldo político. Como consecuencia, esto llevó a explorar, probar y finalmente diseñar y aplicar nuevos instrumentos.

El costo de las encuestas realizadas es muy bajo, en comparación con las encuestas de origen y destino. La plataforma web tuvo un costo de US $\$ 50$ cada tres meses de uso, a lo que hay que sumar sueldos de seis meses para 8 personas semicalificadas, con dedicación de medio tiempo a la actividad de relevamiento, y de dos personas calificadas de tiempo completo. El trabajo de elaboración, debate y pruebas piloto de cuestionario es difícil de mensurar en términos económicos y lo absorbe el equipo de investigación más antiguo. El trabajo de campo complementario, implica gastos en transporte y, en los casos en que no funcionó el wi-fi, se sumó la impresión de formularios y la compra masiva de lapiceras, además del trabajo de carga de datos (Aón, 2017).

También es necesario señalar la importancia de celebrar convenios con actores y sectores que tienen intereses comunes con el observatorio, en materia de datos de transporte y movilidad. En este caso, el trabajo histórico y cotidiano iniciado por el observatorio con el municipio, fue derivando en los diferentes convenios con la secretaría de gestión pública del municipio, así como también con Uta-La Plata. Estos convenios onerosos impulsan el trabajo de construcción de encuestas añadiendo posibilidades económicas de mayor despliegue para los operativos. Sin embargo, se presenta el desafío de hacer converger las agendas de investigación de grupos e individuos con los plazos y tiempos establecidos por la demanda, lo cual requiere habilidades de organización y gestión desde el observatorio. 


\section{Sobre el trabajo en equipo}

El proceso de construcción de metodologías requiere un impulso permanente. Desde el Observatorio de movilidad fue, y es desde siempre, una necesidad imperiosa contar con nueva información de movilidad. Evitando trabajar con datos antiguos proyectados y poniendo en valor los avances metodológicos orientados a construir de manera permanente información de calidad para la investigación y para la planificación. Este precedente, combinado con un contexto de escasez de recursos económicos para realizar relevamientos y la convergencia de cierta masa crítica de investigadores, fundamentalmente de becarios, ha generado la ocasión de producir relevamientos ejecutados colectivamente.

El proceso de construcción colectiva fue pensado en el marco de proyectos de investigación que involucran los intereses individuales de los becarios e investigadores y que alcanzan a la ciudad de La Plata. Este punto de partida permitió el involucramiento de cada persona en un proceso grupal y colectivo, sobre el cual se fueron pautando conceptos, diseños de instrumentos, prueba y análisis de errores, para continuar un proceso de evolución basado en la praxis y la reflexión.

Este intenso proceso de trabajo del equipo, articulando objetivos individuales con objetivos comunes, fue una gran clave del proceso de aprendizaje y construcción colectiva de conocimiento sobre la ejecución de encuestas en atractores de viaje. Esta construcción colectiva fue conduciendo las sucesivas etapas y ajustes, las redefiniciones y mejoras en las tareas previas, de campo y de procesamiento.

La experiencia acumulada del equipo es, como señalamos anteriormente, difícil de mensurar (incluso invaluable). La evolución de los formularios, así como la instalación de nuevos sistemas de georreferenciación, son producto del intercambio de jóvenes y experimentados, cada uno aporta en términos de su disciplina, experiencia profesional $y$, porque no, vivencias personales.

El constante contacto con el campo también sirve de regulación y corrección para los modos de preguntar, para el uso y procesamiento de los datos, para enriquecer al investigador. El ir y volver del procesamiento nos da capacidad de formular mejores preguntas, de encontrar nuevas y de continuar avanzando en la comprensión de un fenómeno complejo y cotidiano, como es la movilidad.

Proyecto Promocional de Investigación y Desarrollo "Transformaciones de la movilidad y de la accesibilidad Urbana frente a los procesos de localización y relocalización residencial en el partido de la plata 2001- 2011". Directora Laura Cristina Aón. 


\section{Q Bibliografía}

"Alonso, W. (1964). Location and land use. Toward a general theory of land rent. Recuperado a partir de http://www.cabdirect.org/abstracts/19641802976.html

" Aón, L. C. (2013). Encuesta de movilidad urbana para la micro región del Gran La Plata 1993/2003. La Plata.

"Aón, L. C. (2017). Encuesta web de movilidad y análisis de atractores. In A. I. Gutiérrez (Ed.), Manual de recomendaciones sobre el uso de metodologías de estudio (10, pp. 40-46). Ciudad Autónoma de Buenos Aires: EUDEBA.

»Aón, L., Giglio, M. L. \& Cola, C. (2017). Patrones modales de movilidad y desarrollo urbano no planificado en la ciudad de La Plata. Revista Transporte y Territorio, (17), 117-144. http://revistascientificas.filo.uba.ar/index.php/rtt/ article/view/3870

» Aón, L. C., Giglio, M. L., López, M. J., Freaza, N., \& Cola, C. (2017). Observatorio de Movilidad Urbana Gran La Plata TOMO 1. Presentación Institucional Marco Teórico - Metodológico. (L. C. Aón \& M. L. Giglio, Eds.) (10). La Plata: Instituto de Investigación y Políticas del Ambiente Construido.

» Aón, L. C., López, M. J., Freaza, N., Cola, C. A., \& Giglio, M. L. (2017). Observatorio de Movilidad Urbana Gran La Plata. TOMO 2. Atractores de viaje por Salud. (L. C. Aón, M. J. López, \& M. L. Giglio, Eds.) (10). La Plata: Instituto de Investigación y Políticas del Ambiente Construido.

»Aón, L. C., López, M. J., Giglio, M. L., Freaza, N., \& Cola, C. A. (2017). Observatorio de Movilidad Urbana Gran La Plata. TOMO 3. Atractores de viaje: Escuelas. (M. J. López, M. L. Giglio, \& L. C. Aón, Eds.) (10). La Plata: Instituto de Investigación y Políticas del Ambiente Construido.

" Bates, J. J., (1968). Econometric Issues in stated preference analysis. Journal of Transport Economics and Policy 22,59-69.

»Bruton, M. J. (1970). Introduction to transportation planning. Hutchinson, USA

»Lafuente, P. C. (2000). Modelos de Gravitación Comercial: una aplicación al Anuario Comercial de España. Instituto L. R. Klein. Universidad.

»De La Barra, T., Añez J. y Perez, B. (1999). Asignación Probabilística Multimodal. URBANA: Revista Eletrônica do Centro Interdisciplinar de Estudos sobre a Cidade, 4 (25), 67-84. http://190.169.30.98/ojs/index.php/rev_urb/article/view/6625

»Etcheñique, M. (1975). Modelos matemáticos de la estructura espacial urbana: aplicaciones en América Latina. Buenos Aires: Ediciones SIAP.

» EPH (2019). Encuesta Permanente de Hogares 1er trimestre 2019. Gran La Plata. INDEC.

"Gutiérrez, A. (2009). Movilidad o inmovilidad: ¿qué es la movilidad? Aprendiendo a delimitar los deseos. XV Congresos Latinoamericanos de Transporte Público y Urbano. BUENOS AIRES

» INDEC (2010). Censo Nacional de Población, Hogares y Viviendas. Partido de La Plata.

» ITE (1991). Trip Generation Manual. Institute of Transportation Engineers

» Miralles-Guasch, C., \& Cebollada, Á. (2009). Movilidad cotidianaysostenibilidad, una interpretación desde la geografía humana. BAGE: Boletín de la Asociación 
de Geógrafos Españoles, (50),193-216.

»Ortuzar, J. D. and Willumsen, L. G. (1994). Modeling Transport. 2nd Edition, John Wiley and Sons, New York

" PTUMA (2008). Encuesta origen / destino 2008: Movilidad en el Área Metropolitana de Rosario. Programa de Transporte Urbano para Áreas Metropolitanas, Ministerio del Interior y de Transporte, Argentina.

»PTUMA (2009a). Encuesta origen/destino 2009: Movilidaden el Área Metropolitana de Córdoba. Programa de Transporte Urbano para Áreas Metropolitanas, Ministerio del Interior y de Transporte, Argentina.

» PTUMA (2009b). Encuesta origen / destino 2009: Movilidad en el Área Metropolitana de Mendoza. Programa de Transporte Urbano para Áreas Metropolitanas, Ministerio del Interior y de Transporte, Argentina.

»PTUMA (2010a). Encuesta origen / destino 2010: Movilidad en el Área Metropolitana de Tucumán. Programa de Transporte Urbano para Áreas Metropolitanas, Ministerio del Interior y de Transporte, Argentina.

» PTUMA (2010b). Encuesta origen / destino 2010: Movilidad en el Área Metropolitana de Posadas. Programa de Transporte Urbano para Áreas Metropolitanas, Ministerio del Interior y de Transporte, Argentina.

» PTUMA (2012a). Encuesta origen / destino 2012: Movilidad en el Área Metropolitana de Neuquén - Cipolleti. Programa de Transporte Urbano para Áreas Metropolitanas, Ministerio del Interior y de Transporte, Argentina.

» PTUMA (2012b). Encuesta origen / destino 2012: Movilidad en el Área Metropolitana de Resistencia. Programa de Transporte Urbano para Áreas Metropolitanas, Ministerio del Interior y de Transporte, Argentina.

»PTUMA (2012C). Encuesta origen / destino 2012: Movilidad en el Área Metropolitana de Salta. Programa de Transporte Urbano para Áreas Metropolitanas, Ministerio del Interior y de Transporte, Argentina.

" UNC (2000). Encuestas de origen y destino de viajes para la ciudad de Córdoba. Municipalidad de la ciudad de Córdoba y Facultad de Ingeniería de la UNC.

» UNCo (1992). Estudio de demanda de transporte para la ciudad de Neuquén y su región. Facultad de Ingeniería, UNComahue.

»UNCuyo (1986). Estudio de Origen y destino de viajes para la ciudad de Mendoza. Facultad de Geografía, UNCuyo.

»UNCuyo (1998). Estudio de Origen y destino de viajes para el área metropolitana de Mendoza. Facultad de Ingeniería de la UNCuyo y municipalidad de Mendoza.

» UNLP (1993). Estudio de demanda de transporte mediante encuestas de origen y destino de viajes para la Micro región del Gran la Plata. UI6B-IEHAB FAU UNLP y Municipalidad de la ciudad de La Plata.

» UNLP (1998). Encuestas de origen y destino de viajes para el área metropolitana de Neuquén. UNLP - Municipalidad de Neuquén.

" UNR (1993). Estudio de origen y destino de viajes para la ciudad de Rosario y su área metropolitana. Instituto de Transporte, Facultad de Ingeniería.

» UNR (2002). Estudio de origen y destino de viajes para la ciudad de Rosario y su área metropolitana. Instituto de Transporte, Facultad de Ingeniería, UNR.

»UNS (2014). Encuesta origen / destino: Movilidad en el Área Metropolitana de Bahía Blanca. UNS - Municipalidad de Bahía Blanca. 


\section{Laura Cristina Aón / laura.aon@gmail.com}

Arquitecta (UNLP), Magíster en "Paisaje, medio ambiente y ciudad" (Universidad de Chile). Investigador III UNLP GII IIPAC desempeño como director de línea de Investigación "Planificación Territorial y Movilidad". Ha escrito más de 60 artículos acerca de las temáticas de la movilidad, la ciudad, la planificación territorial y participado de diversos eventos científico-académicos como ponente de trabajos de investigación y de consultoría territorial. Actualmente se desempeña como profesor en grado y postgrado de temas urbanos, movilidad y planificación territorial.

\section{María Luciana Giglio / luciana.giglio@gmail.com}

Arquitecta (2013, Universidad Nacional de La Plata), Dra. en Estudios Urbanos (2019, ICO-UNGS) y Becaria de Investigación Posdoctoral CONICET en la Línea 2 del Instituto de Investigaciones y Políticas del Ambiente Construido (CONICET -UNLP). Miembro del Observatorio de Movilidad Urbana de Gran La Plata (OMUGLP). Docente-investigadora FAU UNLP Cat. V. Ha participado como ponente en eventos académicos, talleres y congresos nacionales e internacionales sobre urbanismo, territorio y transporte.

\section{Nadia Silvana Freaza / freazanadia@gmail.com}

Docente de la Facultad de Arquitectura y Becaria de investigación CONICET. Ha participado en proyectos de investigación y proyectos de transferencia en temas de planificación urbana y movilidad. Actualmente se encuentra finalizando la tesis de la Maestría en Paisaje Medio Ambiente y Ciudad, desarrollando un análisis sobre transformaciones paisajistas en contextos de densificación urbana. Ha publicado varios artículos científicos en temas de urbanismo y paisaje.

\section{Cristian Agustín Cola / cristian.lp@live.com}

Arquitecto, Doctor en Arquitectura y Urbanismo por la FAU UNLP, actual becario en investigación postdoctoral de CONICET, miembro del Observatorio de Movilidad del Gran La Plata. Desenvuelve temáticas de planificación del transporte público junto con el desarrollo urbano y consumo energético/emisiones contaminantes.

\section{María Julieta López / m.julieta.lop86@hotmail.com}

ARQUITECTA (2010) egresada de la Facultad de Arquitectura y Urbanismo (FAU). DOCTORA (2016) en Arquitectura y Urbanismo (FAU UNLP). De 2009 a 2018 integró el grupo de investigación Movilidad, Territorio y Ambiente del IIPAC (CONICETUNLP). Actualmente es asistente de la Dirección de Planificación y Coordinación Territorial, de la Secretaría de Planificación. Ministerio de Transporte. Ha escrito más de 30 artículos acerca de las temáticas de movilidad, la ciudad, la planificación territorial, las políticas de suelo, el ambiente y el paisaje. 